\title{
The prevalence and severity of early childhood caries in preschool children in the Federation of Bosnia and Herzegovina
}

\author{
Lejla Šačić, Nina Marković, Amra Arslanagić Muratbegović, Amila Zukanović, \\ Sedin Kobašlija
}

Department of Preventive and Pediatric Dentistry, Faculty of Dentistry, University of Sarajevo

\footnotetext{
${ }^{\star}$ Corresponding author: ninamarkovic37@gmail.com Tel.: +38733214249, ext. 107 Fax.: +38733443395
}

Received: 14 January 2016

Accepted: 10 May 2016

Key words: Early childhood caries Oral health - Behavioral factors • Epidemiology.

\begin{abstract}
Objective. The aim of this study was to assess caries prevalence and severity in preschool children in the Federation of Bosnia and Herzegovina. In addition, the relationship between the frequency and clinical types of early childhood caries and behavioral factors, oral hygiene and eating habits were assessed. Subjects and methods. An oral health survey was performed in line with the World Health Organization methodology and criteria. The research consisted of clinical examinations of children to determine dentition status, oral hygiene and severity of caries distribution according to Wyne's classification. Information about behavioral factors was collected by means of a questionnaire administered to parents/guardians. Results. The sample consisted of a total of 165 preschool children aged 3-5 years. Mean dmft (decay, missing, filled teeth index for primary dentition) was 6.79 . The percentage of caries-free children was $17.0 \%$. The results showed a statistically significant correlation between oral hygiene and eating habits, and also the frequency and types of early childhood caries. Conclusion. The present study demonstrates high caries prevalence in preschool children in the Federation of Bosnia and Herzegovina. Community based preventive programs should be developed and urgently implemented, in order to achieve the WHO goals, improve oral and general health, thus improving the quality of life of these populations.
\end{abstract}

\section{Introduction}

Early childhood caries is defined as the presence of one or more decayed (non-cavitated or cavitated lesions), missing (due to caries) or filled tooth surfaces in any primary tooth in a preschool-aged child between birth and 71 months of age $(1,2)$. Caries is a rapid and progressive disease that eventually affects permanent dentition. Early childhood caries is the most common chronic disease in children. The teeth are infected immedi- ately after their eruption (3). The Center for Disease Control and Prevention reports that nowadays dental caries is perhaps the most prevalent infectious disease, five times more common than asthma, and seven times more common than hay fever (4). Although the prevalence of caries has decreased worldwide, the problem persists in many parts of the world in certain segments of society, especially socially vulnerable groups. Common risk factors for this disease are poor nutrition and oral hygiene $(5,6)$. Some 
of the factors that favor the development of early childhood caries are dietary habits, the quality and the frequency of meals, night meals and bottle feeding. Studies show that sugars (sucrose, glucose and fructose), as well as other fermentable carbohydrates, may be triggers for the development of early childhood caries $(7,8)$. Sweetened drinks, fruit juices and soft drinks, with the inadequate re-mineralizing activity of saliva (especially at night due to the reduced flow of saliva), can lead to enamel demineralization and caries lesions (5). Alongside nutrition, oral hygiene is another important etiological factor for early childhood caries. The lack of adequate oral hygiene allows for the prolonged oral presence of fermentable carbohydrates, which enhances the metabolism of cariogenic bacteria from the accumulated dental plaque. Early childhood caries affects the quality of life of affected children and their families, causes dental pain, tooth loss over time and problems eating, sleeping, speaking and socializing. The effects of early childhood caries are predictably severe. Major oral hygiene promotion activities must be focused on good dietary habits, oral hygiene improvement, fluoride program development, and the proper education of youth and future parents.

The aim of this study was to assess caries prevalence and severity in preschool children in the Federation of Bosnia and Herzegovina (FBH). In addition, the relationship between the frequency and clinical types of early childhood caries and behavioral factors, oral hygiene and eating habits was assessed.

\section{Subjects and methods}

\section{Design and setting}

This cross-sectional study of preschool children and their parents or guardians was conducted during 2014. The study consisted of an oral health survey of children, accompanied by a questionnaire for parents or guardians.

\section{Participants}

The total sample was 165 healthy preschool children, living in Sarajevo Canton, which is the largest administrative center and could be considered as representative of FBH. The basic criterion for selection was: preschool children who registered for the first time as patients at the Department of Preventive and Pediatric Dentistry of the Faculty of Dentistry, University of Sarajevo, and the Preschool Dental Office at the Public Health Center of the Sarajevo Canton. The target age was from 3 to 5 years old (mean age was 4.08 years, SD 0.799). Of the total sample, $48.5 \%$ were boys and $51.5 \%$ girls.

\section{Measurements}

The Oral Health Survey was performed in accordance with the WHO guidelines for assessment of dental status (9). Wyne's classification was used to determine the progression and severity of the disease.

There are three types of early childhood caries, according to Wyne's Classification (10): Type 1 (mild to moderate) - the existence of isolated carious lesions involving molars and/or incisors. Type 2 (moderate to severe) - labial-lingual carious lesions affecting maxillary incisors, with or without molar caries, depending on the age of the child and the stage of the disease. Mandibular incisors are unaffected. Type 3 (severe) carious lesions affecting almost all the teeth including the mandibular incisors.

Possible behavioral factors that might lead to the presence and progression of early childhood caries were assessed by a survey questionnaire, administrated to parents/ guardians, who accompanied their children to the dentist. The questionnaire contained 
questions on demographics and also about general medical history, dietary intake, fluoride programs, oral hygiene habits and previous dental care. Parents were questioned about the total number of meals per day (used as an ordinal variable, ranked from higher to lowest frequency), the total number of night meals (used as an ordinal variable, ranked from higher to lowest frequency), the age of the child when the practice of oral hygiene began (used as ordinal variables with appropriate age ranks applied), whether fluoride toothpaste was used for daily brushing of teeth (used as ordinal variables with ranked answers from lower (e.g. almost never), medium (1-2 times per week use) and higher (used daily after meals (3-5 times per day)), whether fluorides protect the teeth from development of caries (observed as an ordinal variable, from lowest to highest implication to oral health protection (as: don't know, disagree, agree, strongly agree), and whether the child was fed by bottle or breastfed (used as ordinary variables, ranked per month of infant feeding). The offered answers were ranked in order to assess the degree of behavioral influence on oral health from highest to lowest susceptibility to development of dental caries. Written consent was obtained from parents/ guardians who agreed to participate. All data were kept anonymous and only patient gender and age were noted.

\section{Ethics statement}

The study protocol was reviewed and approved by the Local Ethics Committee of the Faculty of Dentistry, University of Sarajevo.

\section{Statistical analysis}

The results were presented by means of descriptive statistics (frequency distribution, percentage, mean $\pm \mathrm{SD}$, Median, Range, Minimum, Maximum and Percentiles). Sta- tistic differences between studied groups were tested by the Kruskal Wallis test with the Sidak post- hoc test to note exact differences. The Spearman correlation was used to assess the relationship between $\mathrm{dmft}$, Wyne types and oral hygiene and dietary habits. The level of significance was set at $\mathrm{p}<0.05$. The statistical packages Microsoft Office Excel 2007 and IBM SPSS Statistics 21 were used for data analysis.

\section{Results}

In accordance with the WHO Oral Health Surveys prescribed methodology, the prevalence of dental caries was recorded and analyzed using the dmft index (9). The results of descriptive statistics show that $50 \%$ of subjects had dmft values in a range of 2 to 10.5 , $25 \%$ of subjects had the lowest $\mathrm{dmft}$ values in a range of $0-2$, and $25 \%$ of subjects had the highest values in a range of 10.5 to 20 . The median was 7 and the mean $\mathrm{dmft}$ was 6.8 (SD 5.25). The percentage of children without caries was $17 \%$. The percentage of caries free children decreased with age from $41.3 \%$ of children aged 3 to $13.6 \%$ at age 4 and only $1.7 \%$ at age 5 . The percentage of untreated decay was highest among five year olds (98.3\%). The caries experience, in study groups and in the total sample, presented as $\mathrm{dmft}$ index values and distribution of its components is given in Table 1 .

The severity of caries distribution in preschool children using Wyne's classification is shown in Table 2.

Despite the high values of untreated caries in the examined population, most of the children scored as Wyne type 1. The Kruskal Wallis test was used to test differences in $\mathrm{dmft}$ values between age groups. A significant difference was observed (Chisquare $=14.431, \mathrm{p}<0.001$ ). The Sidak post hoc test confirmed a significant difference between children aged 3 and $5(\mathrm{p}<0.001)$, whereas $\mathrm{dmft}$ was lower in children age 3 
Table 1 Summary of decay experience in preschool children, presented in the total sample by age groups

\begin{tabular}{lllll}
\hline Age grups (years) & $\mathrm{dmft}($ mean $\pm \mathrm{SD})$ & Decay teeth $($ mean $\pm S D)$ & Missing teeth $($ mean $\pm S D)$ & Filled teeth $($ mean $\pm S D)$ \\
\hline $3(n=46)$ & $5.1 \pm 5.8$ & $4.7 \pm 5.6$ & $0.0 \pm 0.0$ & $0.3 \pm 0.8$ \\
$4(n=59)$ & $6.5 \pm 5.1$ & $6.0 \pm 5.2$ & $0.1 \pm 0.3$ & $0.5 \pm 1.1$ \\
$5(n=60)$ & $8.3 \pm 4.4$ & $6.9 \pm 4.2$ & $0.1 \pm 0.4$ & $1.3 \pm 2.4$ \\
\hline Total $(n=165)$ & $6.8 \pm 5.2$ & $5.9 \pm 5.1$ & $0.1 \pm 0.3$ & $0.7 \pm 1.7$ \\
\hline
\end{tabular}

$\mathrm{dmft}=$ decay missing filled teeth for primary dentition.

Table 2 Severity of caries distribution in preschool children by Wyne's classification according to age groups

\begin{tabular}{llllll}
\hline Age groups (years) & Caries free $\mathrm{n}(\%)$ & Wyne Type $1 \mathrm{n}(\%)$ & Wyne Type $2 \mathrm{n}(\%)$ & Wyne Type $3 \mathrm{n}(\%)$ & Total $\mathrm{n}(\%)$ \\
\hline $3(\mathrm{n}=46)$ & $19(41.3)$ & $19(41.3)$ & $3(6.5)$ & $5(10.9)$ & $46(100)$ \\
$4(\mathrm{n}=59)$ & $8(13.6)$ & $37(62.7)$ & $10(16.9)$ & $4(6.8)$ & $59(100)$ \\
$5(\mathrm{n}=60)$ & $1(1.7)$ & $43(71.7)$ & $13(21.7)$ & $3(5)$ & $60(100)$ \\
\hline Total $(\mathrm{n}=165)$ & $28(17)$ & $99(60)$ & $26(15.8)$ & $12(7.3)$ & $165(100)$ \\
\hline
\end{tabular}

Caries free=percentages of examinees without caries; Wyne Class $1=$ mild to moderate progression of disease; Wyne Class $2=$ moderate to severe progression of disease; Wyne Class $3=$ severe progression of disease.

by 3.28 respectively. There were no statistically significant differences between children aged 3 and 4 , and 4 and 5 ( $\mathrm{p}>0.05$ ). Differences between age groups and Wyne class scoring were also tested with the Kruskal Wallis test. A significant difference was observed between the tested variables (Chisquare $=13.448, \mathrm{p}<0.001$ ). Differences were found by the Sidak post hoc test between age groups 3 and $5(\mathrm{p}<0.01)$. Post hoc testing did not confirm any significant difference between the other tested aged groups ( $p>0.05$ ).

A total of 165 parents/guardians were surveyed, $90.9 \%$ of them indicated that caries may be prevented by good oral hygiene. Almost the same percentage of parents knew that fluoride can protect teeth from caries $(67.3 \%)$ and were using fluoride toothpaste for brushing (68.5\%). A high percentage of parents/guardians (95.2\%) indicated the use of a toothbrush and toothpaste for brushing their children's teeth every day. Seventy percent of parents thought that caries could be prevented by proper nutrition, $15.2 \%$ of children still had night meals and sometimes used a feeding bottle.

Spearman's correlation was conducted between the dmft index and Wyne types, and the number of meals per day, the frequency of intake of sweets, the number of times they brush their teeth each day, the frequency of dentist visits, the frequency of night meals, the age when oral hygiene practices began, and their attitude to fluorides. A statistically significant positive correlation was observed between $\mathrm{dmft}$ and the age of beginning oral hygiene $(\mathrm{r}=0.363, \mathrm{p}=0.001)$, the attitude towards the caries preventive effects of fluorides $(r=0.29, p=0.000)$ and the Wyne class and the age of beginning oral hygiene $(\mathrm{r}=0.28, \mathrm{p}=0.005)$.

\section{Discussion}

The mean value of $\mathrm{dmft}$ in the population studied is quite high according to WHO standards (11). A high percentage of preschool children had untreated carious lesions. Comparing these results with previous studies from the country, it is evident that the epidemiology of the studied population has not changed. In the survey conducted in 1999 the mean caries prevalence in preschool children in Bosnia and Herzegovina $(\mathrm{BH})$ was reported as $\mathrm{dmft} 6.87$ and the percentage of untreated caries $90.7 \%$ 
(12). In 2001 the mean dmft was 6.8 and the percentage of untreated caries was $87.7 \%$ (13), and the latest available data showed no improvement, reporting a mean $\mathrm{dmft}$ of 6.7 and the percentage of untreated caries as $88.8 \%$ in 2004 (14).

The prevalence of early childhood caries is different around the world. In Croatia $30 \%$ of children aged 3-5 develop early childhood caries (9). Similar results were reported for some parts of Serbia (30.5\%) (15), and in Bulgaria (32\%) (16). The lowest prevalence of early childhood caries was reported in Canada, at 5\% (17).

The classification of early childhood caries by Wyne (10) gives a better insight into the severity of the disease and the extent of the problem for health care workers. The distribution and severity are in line with the general prevalence of early childhood caries for the investigated population. Good oral hygiene, alongside other preventive measures, is the most economical, most accessible and most practical way to prevent tooth decay. A high percentage of parents/ guardians, 90.9\%, indicated that caries could be prevented by good oral hygiene, but epidemiological findings revealed that this was not properly applied in practice. The American Academy of Pediatric Dentistry (AAPD) recommends that oral hygiene should begin with the eruption of the first tooth, and that parents should assist children in tooth brushing until the age of 10 (18). Most of the respondents indicated brushing their children's teeth at least twice a day, but it is probable that in practice oral hygiene is not carried out thoroughly, which results in a high prevalence of dental caries. The importance of the frequency of tooth brushing, and the importance of parental involvement has been emphasized in many studies (19). In the present study, $70.3 \%$ of parents were aware of the importance of nutrition for good oral health, which is quite good progress compared to research from 2001, where only $25 \%$ of parents recognized the importance of nutrition (13). Besides the fact that most parents were aware of the importance of nutrition, the results showed that almost all children consumed sweet drinks and foods at least once a day. The importance of the frequency of consumption of less sweet food and fewer sugary drinks has been known for a long time. A study conducted in 1954 reported that sugar consumption less than 4 times per day during meals, does not favor the development of dental caries (19). The Scandinavian countries, which are very successful in preventive care and caries reduction in children of pre-school age, have a usual "day for sweets", thus their consumption is minimized to a weekly level; e.g. in Denmark $45 \%$ of children and in Finland $21 \%$ of children only consume sweets on a certain day of the week (19). Night meals are a very important risk factor for development of early childhood caries. The recommendation of AAPD is to stop breastfeeding when the child is one year old (18). The WHO recommends breastfeeding until the child is two years old, with the introduction of supplementary feeding at six months (19). Parental attitudes regarding this specific issue were in line with these guidelines, although most of the parents agreed with the AAPD recommendation to stop breastfeeding when the child is one year old. In comparison with previous investigations, levels of knowledge and awareness have been obviously raised, but practice of good oral health habits is still lacking.

\section{Conclusion}

The present study provides evidence of the relatively high caries prevalence in preschool children in the $\mathrm{FBH}$, with mean dmft of 6.7 and the small percentage of children without caries (17\%). The distribution of Wyne types revealed the presence of a higher progression and severity of early childhood 
caries among the youngest examinees. The percentage of 3 year-olds classified as Wyne type 3 indicates that the focus on preventive measures has to be placed on education and motivation for proper oral hygiene and good nutrition practices as early as possible. A higher frequency of sugar intake, late onset of oral hygiene practices, lack of fluoride exposure and lack of knowledge about fluoride impact to oral health protection were behavioral factors related to higher values of dmft and scoring of Wyne types in examined population. The positive parental attitude regarding behavior and practice was not in line with the prevalence and severity of the disease. Community-based preventive programs have to be developed and implemented urgently in order to achieve the WHO goals and to improve oral health, health in general and the quality of life.

\section{What is already known on this topic}

There has been a considerable decrease in caries prevalence in many European countries in recent decades. Caries epidemiology involves the study of variables that may influence its occurrence and severity, including behavioral factors, such as knowledge of and attitude towards dietary and oral hygiene habits. Recent studies have shown the complex interaction of these factors and caries in preschoolers. The last epidemiological data on caries in preschool children in FBH were assessed in 2004.

\section{What this study adds}

It is the first epidemiological study in FBH where the severity of early childhood caries has been assessed and analyzed. In addition, this study assesses the behavioral variables and interaction with early childhood caries experience and severity in children in FBH.

Author's contributions: Conception and design: LS and NM. Acquisition, analysis and interpretation of data: LS, NM and AZ; Drafting the article: LS and NM; Revising it critically for important intellectual content: NM, AAM and SK; Approved final version of the manuscript: LS, NM, AAM, AZ and SK.

Conflict of interest: The authors declare that they have no conflict of interest.

\section{References}

1. Drury TF, Horowitz AM, Ismail AI, Maertens MP, Rozier RG, Selwitz RH. Diagnosing and reporting early childhood caries for research purposes. A report of a workshop sponsored by the National Institute of Dental and Craniofacial Research, the Health Resources and Services Administration, and the Health Care Financing Administration. J Public Health Dent. 1999;59(3):192-7.

2. Kaste LM, Drury TF, Horowitz AM, Beltran E. An evaluation of NHANES III estimates of early childhood caries. J Public Health Dent. 1999;59:198200.

3. Douglass JM, Douglass AB, Silk HJ. A practical guide to infant oral health. Am Fam Physician. 2004;70(11):2113-20.

4. Hale KJ; American Academy of Pediatrics Section on Pediatric Dentistry. Oral health risk assessment timing and establishment of the dental home. Pediatrics. 2003;111(5 Pt 1):1113-6.

5. Seow WK. Biological mechanisms of early childhood caries. Community Dent Oral Epidemiol. 1998;26(1 Suppl):8-27.

6. Lulić-Dukić O, Jurić H, Dukić W, Glavina D. Factors predisposing to early childhood caries (ECC) in children of pre-school age in the city of Zagreb. Coll Antropol. 2001;25:297-302.

7. Douglass JM. Response to Tinanoff and Palmer: Dietary determinants of dental caries and dietary recommendations for preschool children. J Public Health Dent. 2000;60(3):207-9.

8. Paes Leme AF, Koo H, Bellato CM, Bedi G, Cury JA. The role of sucrose in cariogenic dental biofilm formation--new insight. J Dent Res. 2006;85(10):878-87.

9. World Health Organization (WHO). Oral Health Surveys: Basic methods. 4th edition. Geneva: WHO; 1997.

10. Wyne AH. Early childhood caries: nomenclature and case definition. Community Dent Oral Epidemiol. 1999;27(5):313-5.

11. Hobdell M, Petersen PE, Clarkson J, Johnson N. Global goals for oral health 2020. Int Dent J. 2003;53(5):285-8.

12. Kobašlija S, Maglajlić N, Huseinbegović A, Tahmiščija H. The Prevalence of dental Caries in Children. Acta Stomatol Croat. 2000;34(1):83-5.

13. Huseinbegović A. Social and medical aspects of primary dentition caries in urban conditions. [master's dissertation]. Sarajevo: University of Sarajevo, School of Dentistry; 2001.

14. Marković N, Arslanagić Muratbegović A, Kobašlija S, Bajrić E, Selimović-Dragaš M, Huseinbegović A. Caries prevalence of children and adolescents in Bosnia and Herzegovina. Acta Med Acad. 2013;42(2):108-16. 
15. Tušek I, Carević M, Tušek J. The Presence of early childhood caries among members of different ethnic groups of The South Bačka District [in Serbian].Vojnosanit Pregl. 2012;69(12):1046-51.

16. Markova N. Early childhood caries-caries infantiae. [PhD dissertation]. Sofia: University of Sofia; 2003.

17. Colak H, Dülgergil CT, Dalli M, Hamidi MM. Early childhood caries update: A review of causes, diagnoses, and treatments. J Nat Sci Biol Med. 2013;4(1):29-38.

18. American Association of Pediatric Dentistry (AAPD). Early childhood caries: unique challenges and treatment options. Pediatr Dent. 2000;22:21.

19. World Health Organization (WHO). Global strategy for infant and young child feeding. Geneva: WHO; 2003. 